\title{
An Integrated Dynamic Simulation Platform for Assistive Human-Robot Interaction: Application to Upper Limb Exosuit
}

\author{
Ratna Sambhav, Shreeshan Jena, Ankit Chatterjee, Sitikantha Roy*, Shubhendu Bhasin, \\ Sushma Santapuri, Lalan Kumar and Suriya P. Muthukrishnan
}

\begin{abstract}
Soft exosuits are wearable robotic devices that assist or enhance the human muscle performance. $A$ human machine interface simulation platform based on MATLAB-OpenSim interface is developed in this paper for closed loop dynamic simulation with feedback control strategy and to study its effect on human physiology. The proposed simulation model is based on Computed Muscle Control (CMC) algorithm and is implemented using the MATLAB -OpenSim interface. A Gravity Compensation (GC) controller has been implemented on the external device and the resulting decrease in the physiological torques, muscle activations and metabolic costs during a simple load lifting task with two different speeds is investigated.
\end{abstract}

Index Terms-Exosuit, Human in-loop biomechanics, Human-Machine Interaction, Musculoskeletal simulation, Assistive device

\section{Introduction}

$\mathrm{E}_{\mathrm{in}}^{\mathrm{x}}$ XOSKELETONs and exosuits are the terms used interchangeably to refer to a class of wearable assistive devices which work in tandem with the human body to provide assistance. The assistance provided by these human joint force amplifiers can augment, reinforce or even restore human performance. Muscle strength augmentation for workers or soldiers [1], assistance to elderly generation in ADLs [2], muscle performance restoration in paraplegic patients [3], are

Manuscript received on The present work was supported by the Joint Advanced Technology Centre, Defence Research and Development Organisation (Project No. RP03830G).

${ }^{*} S$. Roy is with the Department of Applied Mechanics, Indian Institute of Technology Delhi, New Delhi -110016, India (e-mail: sroy@am.iitd.ac.in).

S. Santapuri, R. Sambhav and S.Jena are with the Department of Applied Mechanics, Indian Institute of Technology Delhi, New Delhi 110016, India (e-mail: ssantapuri@am.iitd.ac.in, sambhav.ratna@gmail.com, shreeshan24@gmail.com)

S. Bhasin, L. Kumar and A. Chatterjee are with the Department of Electrical Engineering, Indian Institute of Technology Delhi, New Delhi - 110016, India. (e-mail: Shubhendu.bhasin@ee.iitd.ac.in, Ikumar@ee.iitd.ac.in, chatterjee.ankit835@gmail.com)

S. P. Muthikrishnan is with the Department of Physiology, All India Institute of Medical Sciences, New Delhi - 110029, India. (e-mail: dr.suriyaprakash@aiims.edu) few of the many applications of these devices. A review of existing devices and research approaches suggests that the exosuit design depends on some crucial developmental aspects: the user intention estimation, assistance torque estimation, and the comfort of force transmission [4-7]. As the experimental evaluation of these aspects on a physical setup can be tedious and accompanied by safety concerns, there is a requirement for the development of an integrated simulation framework where a virtual human model coupled with an external augmentation device can be tested and subsequently optimized. Prior simulation also enables device optimization based on the parameters that are difficult to measure experimentally, such as the interaction forces, joint reaction forces, etc. The simulation framework requires an integrated environment where along with musculoskeletal dynamics, intention estimation using a brain control interface (BCI), controller implementation and actuator modules can be collated to study the resulting physiological parameters on the virtual human model. Finite element analysis (FEA) has been utilized in recent studies for estimating the user comfort, and can also be conceptualized as an additional module within the framework [7]. The initial challenges with the development and implementation of musculoskeletal models have been outlined in recent studies [8$10]$.

In recent literature, Zhang et al. employed an inverse dynamics and optimization-based technique using the Anybody Inc. software to observe the effects of different assistive strategies on the reduction in physiological torque and muscle impulse [7]. They utilized static optimization technique to calculate the muscle activations and the interaction forces at all the contact points between the exoskeleton and human body [7]. Although this approach is computationally efficient, the absence of a feedback correction term makes it sensitive to even slight errors in the predicted muscle activations. A feedback method is adopted by Stollenmaier et al. where open loop and closed motor commands together drive a forward dynamics model [11]. The command generator drives the open loop motor command utilizing the input trajectory, inverse dynamics and optimization steps [11]. Subsequently, the closed loop motor command is obtained after multiplying the error in desired and current values of muscle fiber lengths and contraction velocities with the proportional and derivative feedback terms. This method is motivated by the fact that the ability of a healthy 
human to make smooth and accurate reaching movements with minimal effort, rely on the existence of adaptable internal models of limbs and objects in the brain. Models in the recent literature relate motor commands to changes in object (limb) states (i.e., position and velocity) to influence the signals in a physiologically-accurate and predictable manner [12-14].

In this paper, the position and velocity data of the joint from the forward dynamics are fed back, so that the inverse dynamics-based motor command generator can compensate for the error terms. The open-source platform OpenSim $[15,16]$, that is widely established for musculoskeletal simulations, is implemented in the present simulation framework. MATLAB is the base platform for this simulation framework wherein the multibody dynamics of OpenSim (using the API library) along with the BCI, optimization, control, actuator and physiological modules have been incorporated.

\section{METHOD}

\section{A. Explanation of the Overall System}

The present simulation framework creates a digital model of the human musculoskeletal system integrated with an external assistive device. The overall system architecture is divided into the brain computer interface (BCI), the musculoskeletal biomechanics, actuator and controller modules. This overall architecture is presented in Fig.1, showing the flow of information between each module of the framework.

- Muscle command generator (MCG) calculates the required level of muscle excitation, based on the given reference trajectory and the external assistive forces, and is used to drive the forward dynamics model. The inverse dynamics and musculoskeletal models within the MCG utilize the OpenSim API commands while the static optimization and activation dynamics are carried out entirely in MATLAB algorithm.

- The forward dynamics utilizes OpenSim API commands to determine the joint reaction forces and exosuit-human interaction forces, along with the error between the desired and output joint trajectories, thus refining the accuracy of estimated muscle excitations.

- The controller (MATLAB-based) processes the system dynamics to calculate the desired effort from the external actuator. Additionally, the system dynamics are also used in the actuator model to calculate the required input to the actuator for providing the necessary control.

The implementation of the present system creates a digital human musculoskeletal model and calculates the reduction in the energy requirement of a human with the application of a muscle augmentation exosuit comprising external soft actuators.

\section{B. Muscle command Generator (MCG)}

The purpose of MCG is to use the error between the desired and actual trajectories to calculate the required excitations in the six muscles such that the trajectory error is also minimized. The MCG also utilizes the position and velocity feedback from the simulated output trajectory. Inverse dynamics calculates the joint moments from the input kinematics, followed by the static optimization to solve for the muscle redundancy problem and obtain the muscle activations. Finally, the activation dynamics equations are used to calculate the corresponding muscle excitation values.

The MCG functionality is explained using the following:

Inverse Dynamics: The desired joint trajectory as well as the error between the simulated and desired trajectories is used to calculate the joint moments (in the presence or absence of an external force) such that the joint torque,

$$
\tau=M \ddot{\theta}-G(\theta)-C(\theta, \dot{\theta})-A(\theta, \dot{\theta}, t)
$$

where, $A(\theta, \dot{\theta}, t)$ term represents any externally applied force or torque corresponding to the position $(\theta)$, velocity $(\dot{\theta})$ and time $(t)$. The terms $G(\theta)$ for gravity and $C(\theta, \dot{\theta})$ for centripetal and Coriolis, are calculated from the current values of $\theta$ and $\dot{\theta}$. However, in the inertial term $M \ddot{\theta}$, the acceleration at the joint is the sum of desired acceleration $\left(\ddot{\theta}_{\text {des }}\right)$ and the feedback error terms such that,

$$
\ddot{\theta}=\ddot{\theta}_{d e s}+k_{p}(\Delta \theta)+k_{v}(\Delta \dot{\theta})
$$

, where $k_{p}$ and $k_{v}$ are the feedback gains for the position and velocity errors respectively. Equation (2) allows for the compensation of the errors in position and velocity of the joints. In case of assistance, the term $A(\theta, \dot{\theta}, t)$ becomes non-zero, resulting in reduced physiological torque.

Muscle Model: The musculoskeletal model comprises a forward simulation of the arm26 musculoskeletal model, and the Hill-type muscle model has been implemented to execute the resulting joint motion from the input controls. The Hill-type muscle model has been utilized for the forward simulation within the present framework to present the net force in the muscles as

$$
F=F_{m}\left[f(l) f(v) a(t)+f_{p}(l) \cos (\varnothing(t))\right]
$$

, where, $F_{m}$ is the maximum isometric force of the muscle, $f(l)$, $f(v)$ and $f_{p}$ are the generic force-length, force-velocity and passive elastic force-length curves $[17,18]$. The activation input is represented as $a(t)$ and the muscle pennation angle by $\varnothing(t)$.

Optimization: The individual muscle forces are calculated by optimizing the distribution of the activation signals $\left(a_{i}\right)$ among the muscles. The optimization function uses the objective function $\mathrm{J}$ of the form

$$
J=\sum_{i=1}^{6} a_{i}^{2}
$$

, where, $i$ represents the muscles (Biceps Long, Biceps Short, Brachialis, Triceps Long, Triceps Medial and Triceps Lateral), involved in the simulation. The optimization function utilizes the constraints by equating the torque calculated from the inverse dynamics to the torque calculated as a product of the 
individual muscle forces and respective moment arms about the joint. Optimization was done using fmincon function in MATLAB, where equation (4) was set as the minimization function, subject to the equality constraints obtained from equation (1) and the following:

$$
\tau=\sum_{i=1}^{6} d_{i} F_{i}
$$

and the inequality constraints

$$
0 \leq a_{i} \leq 1
$$

, where $\tau$ is the elbow physiological torque, $d_{i}$ is moment arm of the muscles about elbow joint, $F_{i}$ is the force exerted by individual muscles and $a_{i}$. is the activation corresponding to the $i$ th muscle.

Activation Dynamics: The forward dynamics simulation in the system framework requires the muscle control signals (excitations) as input. The individual muscle excitations are calculated from their activation values using the activation dynamics equation [13] shown below:

$$
\frac{d a}{d t}=\frac{u-a}{\kappa_{a}(a, u)}
$$

, where, $\kappa_{a}(a, u)$ is the time constant whose magnitude depends upon whether the muscle activation is increasing or decreasing.

$$
\kappa_{a}(a, u)=\left\{\begin{array}{cl}
\kappa_{a c t}(0.5+1.5 a) & , u>a \\
\kappa_{\text {deact }} /(0.5+1.5 a) & , u \leq a
\end{array}\right.
$$

The parameters $a$ and $u$ are the muscle activations and excitations, respectively. The parameters $\kappa_{\text {act }}$ and $\kappa_{\text {deact }}$ denote the activation and deactivation time constants respectively.

\section{Control system}

A gravity compensation (GC) based control strategy is implemented in the assistive device. In the present study, the assistive torque at the joint is directly proportional to the angle between the forearm and the direction of gravitational force at the elbow joint. The GC controller compensates for the gravity dependent component of the torque acting at the elbow joint, given by:

$$
\tau=m g l_{c} \sin \theta+M g l_{l} \sin \theta
$$

, where $\theta$ is the angle between forearm and the vertical line, $m$ is forearm mass, $M$ is mass of extra load, $l_{c}$ is distance of center of mass of forearm from the elbow joint, and $l_{l}$ is the distance of extra load from the elbow joint. The GC strategy is simiar to that adopted by Dinh et al. [3]. In a physical exosuit, $\theta$ can be obtained from the inertial measurement units (IMUs) attached to the forearm. However, in the simulation environment this information is taken from the measured output elbow joint angle.

\section{Actuator module}

This module is used to calculate the forces in the extensor and flexor cables such that the desired torque can be transmitted to the elbow joint. In the physical exosuit, tension in the flexor and extensor cables will be generated by the motor but in the case of simulation, these forces are directly generated using the path actuators (OpenSim API). The actuator module is based on the current value of moment arms of flexor-extensor cables, in addition to the magnitude and direction of the desired torque (to be transmitted to the elbow joint), and computes the value of the tension required in the cables. These forces in the simulation are directly applied to the path actuators in the forward dynamics block.

For Actuator Torque $>0$ :

$$
\begin{gathered}
T_{1}=\tau_{a} / r_{1}, \\
T_{2}=0,
\end{gathered}
$$

For Actuator Torque $<0$ :

$$
\begin{gathered}
T_{1}=0, \\
T_{2}=\tau_{a} / r_{2},
\end{gathered}
$$

, where $T_{1}$ is the force in flexor cable, $T_{2}$ is the force in extensor cable, $r_{1}$ is the moment arm of flexor cable, and $r_{2}$ is the moment arm of extensor cable.

\section{E. Simulation Platform}

A MATLAB based platform is created and the OpenSim functionalities are accessed from MATLAB, enabling the use of both MATLAB and OpenSim. Using the OpenSim API, forward and inverse dynamics of the musculoskeletal system are performed and the calculations involving controller, actuator and muscle activation are carried out in MATLAB. The simulation platform is versatile and provides easy integration with other software, so that in future other utilities such as brain machine interface and finite element analysis for study of force interaction can be easily interfaced into the simulation. The forward dynamics tool of OpenSim, integrates activation dynamics, muscle contraction dynamics and multibody dynamics. Accordingly, the input to the forward dynamics is the muscle excitation and the output is the resulting joint kinematics. One of the basic elements of the OpenSim based simulation is a musculoskeletal model and its corresponding state variables. The musculoskeletal model is an interconnected multibody structure of bones, connected through joint definitions, with muscles routed over them, functioning as force generating elements. The state variables, as the name suggests, consist of all the numerical values regarding configuration of different joints, muscle parameters, etc., of the musculoskeletal model.

\section{F. Physical Model and its Digital Counterpart}

Fig.2(a) illustrates through CAD model, the physical design of the exosuit on a dummy human model. Exosuit design consists of the following units: (i) wearable fabric and straps, (ii) actuator unit, (iii) controller unit, (iv) cable routing, and (v) 
battery pack. As shown in Fig. 2(a), the actuator and controller units with battery are placed on the back of the human. Bowden cables (agonistic and antagonistic) are routed along the lines of minimum extension, covered with sheath, to the arm straps. The Bowden cables are attached between the upper arm and forearm straps to form the flexor and extensor pairs. Based on the torque and rotational direction of motor, tension is generated in the flexor or extensor cable.

For its digital counterpart, a right upper body model 'arm26' has been used as the musculoskeletal model, representing the digital human, as shown in Fig. 2 (b). This simplistic model provides two degrees of freedom, one at the elbow and another at the shoulder, and the plane of motion is restricted to the sagittal plane. This model has six muscles of the elbow, i.e. biceps long, biceps short, brachialis, triceps long, triceps medial and triceps lateral. The muscles for shoulder joint are omitted, as the present focus is the study of the physiological parameters of the elbow joint with and without assistance.

As a part of the exosuit, the CAD designs of exosuit straps were imported and attached to their appropriate locations over the digital human model. The force transmission system from the motor to the anchorage points are modeled directly as force generating elements between the two straps in the digital model. Path actuators (an actuator class of OpenSim API) have been used as the force generating elements, which apply equal and opposite force on the segments to which they are attached. These path actuators are attached agonistically and antagonistically, in between the upper-arm and forearm straps, as shown in Fig. 2(b). Using the prescribed controller (a controller class of OpenSim), control signals were sent to the path actuators to create the desired level of tension. All the geometrical input parameters of the model are given in Table 1.

\section{G. $B C l$}

Estimating user intended joint motion is a pre-requisite in calculating the optimum level of actuator torque to be generated by the exosuit in order to augment the user's effort. Human motion intention recognition using neural signals like EEG or physiological signals like EMG have several advantages as compared to using physical force signals such as human robot interaction force signals, in terms of latency $(20-100 \mathrm{~ms})$, comfort and safety [19]. The functionality of BCI module in the simulation environment is to test and validate the different methods of desired motion estimation in sync with the other modules of exosuit like controller and actuator by taking data from a pre-recorded EEG dataset. In the current simulation study though, the control system utilizes gravity compensation control which only requires the joint angle data as input, and the BCI module comprises a ready-made reference trajectory as input to the simulation.

\section{H. Control Model}

Unassisted Simulation: The Computed Muscle Control (CMC) algorithm $[10,20]$ has been adopted to generate the muscle excitation signals to follow the desired motion trajectory. CMC based gait simulations are found to provide time histories of muscle activations that were very similar to electromyography recordings [20]. In Fig. 1, the MCG consists of inverse dynamics, static optimization and inverse activation dynamics (excitation calculation from activation). The desired joint speed and joint acceleration vectors are obtained from differentiation of position data and fed into the simulation model. The current joint position and speed vectors are taken as feedback which are then used to compute the position and speed errors. A PD controller is used to generate the desired joint acceleration. In the inverse dynamics block, the joint torques are estimated based on the desired joint acceleration and current joint position and speed. Static optimization utilizes the desired joint torque and current muscle states as input and to calculate the muscle activations. The activation dynamics processes the muscle activations to obtain the corresponding muscle excitations to drive the forward dynamics simulation.

Assisted Simulation: The simulation with an external assistive device (modeled as the actuators in the modified model) function on the signals from the control scheme. The modifications to the existing CMC architecture [20] has been shown in Fig.1. The control block uses the kinematic information of the 'assisted' joint as input, and based on the selected control scheme, presents the assistance torque as output. This assistance torque has to be generated by the exosuit actuators about the joint. This assistance torque in turn, translates to the tension in the flexor and extensor cables. The actuator block computes the magnitude of the required tension in the cable, using the moment arm and direction of motion from the current state variables of the model. The cable forces from the actuator block are fed directly to the path actuators, for the forward dynamics simulation. The application of these assistive forces is hypothesized to reduce the human muscle effort.

\section{SIMULATION}

The goal of the present study is to develop an integrated musculoskeletal simulation platform to comprehend the physiological assistance provided by an exosuit. The simulation was repeated for two conditions of the elbow flexion: (a) a fast and (b) slow flexion motion, with and without assistance from the exosuit. In the current simulation study, we constrained our analysis to elbow joint only, and locked the shoulder degree of motion, so as to obtain intuitive results which can be easily interpreted. Following are the parameters varied in different simulation iterations:

Payload in Hand: The response of the muscles can be observed by changing the physical parameters of the model. An external mass has been attached at a distance of $29 \mathrm{~cm}$ from the elbow joint, as shown in the Fig. 2. The mass of this load is varied ( 0 $\mathrm{kg}, 2 \mathrm{~kg}, 5 \mathrm{~kg}$ ) to observe the rise in muscle activation patterns and subsequently, the reduction in the muscle activity after incorporating actuator assistance.

Reference Trajectory: The elbow joint of the simulation model undergoes motion from an initial angular position of $0^{\circ}$ to a final position of $90^{\circ}$, following a minimum jerk trajectory path for a specified time duration. As shown in Fig.3, two types of motion are used for simulation, one which achieves this trajectory in $1 \mathrm{~s}$, and the other which achieves it in $2 \mathrm{~s}$. Throughout the simulation, the shoulder joint is locked at a $0^{\circ}$ orientation. The two input reference trajectories for achieving the desired orientation are shown in the Fig.3. 


\section{Results}

The developed simulation framework presents the muscle activations, physiological torque, human-exosuit interaction force, elbow joint reaction force and metabolic cost as results of the simulation.

\section{A. Muscle Activations}

The computed muscle activations (Eq. (4)) are presented in the Fig. 4 (A-B) for the high-speed trajectory with and without assistance. The results depict the relative contribution of each muscle towards the desired motion. In the case of the high-speed trajectory, the root mean squared (RMS) value of muscle activities averaged over the three biceps muscles, decreases by $73.72 \%, 67.59 \%$ and $55.12 \%$ respectively for $0 \mathrm{~kg}, 2 \mathrm{~kg}$ and 5 $\mathrm{kg}$ payloads. Comparatively, for the slow speed trajectory, the decrease observed are $74.2 \%, 80.9 \%$, and $76.7 \%$. The muscle activations of the triceps group in case of unassisted motion present a magnitude of about 0.01 , irrespective of the load. However, for the condition with assistance the triceps group shows increased activations. For the high-speed trajectory, the RMS value of muscle activations averaged over the three triceps muscles increases by $46 \%, 240 \%$ and $455.6 \%$ for $0 \mathrm{~kg}, 2 \mathrm{~kg}$ and $5 \mathrm{~kg}$ loads but for the slow-speed trajectory, the increase observed are $28.8 \%, 23.9 \%$ and $66.8 \%$.

\section{A. Physiological Torque}

The obtained physiological torque increases with higher speed and increase in external mass as shown in Fig. 5 (A). In case of the high-speed trajectory, with assistance, the RMS value of physiological torque decreases by $89.13 \%, 74.05 \%$ and $70.78 \%$ for $0 \mathrm{~kg}, 2 \mathrm{~kg}$ and $5 \mathrm{~kg}$ payloads respectively. For the slow-speed trajectory, after assistance, physiological torque decreases by $80.20 \%, 91.73 \%$ and $87.56 \%$ respectively for 0 $\mathrm{kg}, 2 \mathrm{~kg}$ and $5 \mathrm{~kg}$ mass in hand.

\section{B. Joint Reaction Force}

The OpenSim API commands are utilized to obtain the joint reaction force at the elbow. The resulting joint reaction force at elbow joint is observed to decrease in assisted case compared to the unassisted, as presented in Fig. 7 (A-B). For the high-speed trajectory, $28.4 \%, 25.6 \%$ and $30.83 \%$ of decrease has been recorded respectively for $0 \mathrm{~kg}, 2 \mathrm{~kg}$ and $5 \mathrm{~kg}$ payloads. For the slow-speed trajectory, $17.2 \%, 49.1 \%$ and $51.7 \%$ of decrease has been observed.

\section{Interaction Force}

The normal and shear forces acting on the exosuit strap are calculated by using the OpenSim API commands. Subsequently, the results show that the normal and shear forces applied by the exosuit strap on the forearm increase with the payload mass and remain unchanged for different speeds of elbow joint motion (Fig. 7(C-D)). The peak values of normal forces are $12 \mathrm{~N}, 40 \mathrm{~N}$ and $80 \mathrm{~N}$ respectively for $0 \mathrm{~kg}, 2 \mathrm{~kg}$ and $5 \mathrm{~kg}$ payload mass, whereas the peak values of shear forces are $25 \mathrm{~N}, 80 \mathrm{~N}$ and $160 \mathrm{~N}$, for both high speed and low speed trajectories.

\section{Metabolic Cost}

The OpenSim API functionality is used for calculating the metabolic cost of the musculoskeletal model for the targeted motion [21]. The results of the metabolic cost are presented in the Figure 5(B). The magnitude of resultant metabolic costs of the model depend on work done by muscles and its activation and excitation values. After applying assistance, reduction in RMS value of the metabolic cost is, for high-speed trajectory, $60 \%, 61.83 \%$ and $64.83 \%$ respectively for $0 \mathrm{~kg}, 2 \mathrm{~kg}$ and $5 \mathrm{~kg}$ loads and for low-speed trajectory, it is $44.76 \%, 71.93 \%$ and $75.89 \%$.

\section{DISCUSSION}

The prime motivation of the work is to develop a platform where the crucial parameters involved in the development of an exosuit device can be calculated and efficacy of the control scheme can be assessed. The results from simulation also present insight into parameters that are difficult to obtain experimentally, that is, the joint reaction forces and exosuithuman interaction forces. Thus the simulation presents an opportunity for optimization of the exosuit design based on these parameters. The generalized simulation framework has been applied to an upper limb assistive exosuit. A gravity compensation based assistive strategy has been implemented in the exosuit, and the physiological benefits to an upper body musculoskeletal model, while executing elbow flexion, are studied. The goal of the controller is to provide external assistance such that the biological muscles have to compensate for the inertia component only (Eq. 1), thereby reducing a major portion (gravity component) of the moment required at the elbow joint.

There is a huge reduction in the physiological torque after receiving external assistance, that can be attributed to the GC control strategy compensating for the gravity component of the elbow torque. Further, in case of the low-speed trajectory, the magnitude of acceleration is also lower, i.e., a maximum value of $2.5 \mathrm{rad} / \mathrm{s}^{2}$ (low-speed motion), compared to $8 \mathrm{rad} / \mathrm{s}^{2}$ (highspeed motion). This leads to a lower magnitude of the inertial component for the low speed trajectory, thus increasing the relative proportion of the gravity component in Eq. 1. As a consequence, this results in a higher reduction of the physiological torque for the low-speed trajectory. For higher payload values in the high-speed trajectory (Fig. 5(A)), it can be seen that the physiological torque attains a negative magnitude towards the end of trajectory. This negative magnitude of physiological torque is relatively lower in the case of low-speed trajectory. This is because, towards the end, the deceleration is higher $\left(-8 \mathrm{rad} / \mathrm{s}^{2}\right)$, and thus the torque required at the elbow joint to maintain the desired trajectory is less than that is provided by the exosuit based on GC control strategy. In this situation, the antagonistic muscles (triceps group) present relatively higher activations (Fig. 4 (B)) to counteract the excess of the assistive torque at the elbow joint. The muscle recruitment and the change in their activation levels can be understood by inferring the physiological torque variation (Fig. 4 \& Fig. 5(A)). The Fig. 6(a-b) shows the RMS values of muscle activations for different conditions of loading, speed and assistance. However, even after considering the increase in triceps muscles' activation values, the overall human (muscle) effort decreases with external assistance.

The joint reaction force is another important parameter to quantitatively evaluate the beneficial effects of an assistive device, as an increased joint reaction force can be harmful to the joint. In the case of exoskeleton devices as available in 
literature, the physiological torque as well as the joint reaction force are distributed between the human joint and the corresponding exoskeleton joint. However, the present system framework considers a soft exosuit design that has no rigid external joint. Interestingly, the simulation results illustrate that there is a reduction in the joint reaction force when external assistance is provided (Fig. 7). As shown in Fig. 7, there is a higher reduction in the resultant joint reaction force at elbow (due to assistance from exosuit), with the increase in external load and the speed of motion.

The interaction force between the exosuit strap and the human limb is an important parameter in the design of the exosuit. The dimensions of straps and the padding material to be used in the strap, can be calculated based on the force applied by the strap to the body, in order to bring the contact pressure within the required tolerance [22-24]. The simulation results have important implications for physical design of the suit, and while $80 \mathrm{~N}$ of normal force is well below the maximum tolerable force, $160 \mathrm{~N}$ of shear force may cause the forearm strap to slide over the forearm.

The metabolic cost plots presented in Fig. 5(B) depict a high reduction in metabolic cost, and this can be attributed to the consideration of only six muscles about a single joint. On a physical scale, considering a healthy human, the actual experimental metabolic cost reductions may be of a different magnitude as compared to the simulation results. The validation of these simulation results can be attempted in future research, taking into consideration the appropriate constraints.

The present musculoskeletal model utilized in the simulation framework presents some limitations and may not corroborate clinical data due to the following factors: (a) incorporation of only six muscles about the elbow joint and omitting the muscles at the shoulder and the forearm, (b) the metabolic cost reduction values are those calculated considering these six muscles only, and the actual clinical data can be expected to be different. However, the MATLAB-OpenSim framework provides easy access and utilization of multiple musculoskeletal models. Further, the force interaction between the actuator straps and the human twin may be carried out with the addition of a FEA module.

\section{CONCLUSION}

The simulation framework described in this paper utilizes the best features of OpenSim and MATLAB to develop a system that acts as a digital model of the human physiology. This simulation framework calculates the physiological muscle excitations and the metabolic costs with and without assistance from an external actuator. Further, computing the interaction forces provide sufficient evidence for a proper design of exosuit straps. Overall, the reduction in effort of the human muscle may be estimated using the framework, thereby indicating the efficiency of an exosuit implementing the proposed control strategy.

\section{ACKNOWLEDGMENT}

The present work was funded by the Joint Advanced Technology Centre (JATC), IIT Delhi - Project No. RP03830G.

\section{REFERENCES}

[1] M. Wehner, B. Quinlivan, P. M. Aubin, E. Martinez-Villalpando, M. Baumann, L. Stirling, et al., "A lightweight soft exosuit for gait assistance," in 2013 IEEE Int. Conf. Robot. Autom., 2013, pp. 33623369.

[2] Q. Wu, B. Chen, and H. Wu, "Neural-network-enhanced torque estimation control of a soft wearable exoskeleton for elbow assistance," Mechatronics, vol. 63, p. 102279, 2019.

[3] B. K. Dinh, M. Xiloyannis, C. W. Antuvan, L. Cappello, and L. Masia, "Hierarchical cascade controller for assistance modulation in a soft wearable arm exoskeleton," IEEE Robot. Autom. Lett., vol. 2, pp. 17861793, 2017.

[4] N. Lotti, M. Xiloyannis, F. Missiroli, D. Chiaradia, A. Frisoli, V. Sanguineti, et al., "Intention-detection strategies for upper limb exosuits: model-based myoelectric vs dynamic-based control," in 2020 8th IEEE RAS/EMBS International Conference for Biomedical Robotics and Biomechatronics (BioRob), 2020, pp. 410-415.

[5] D. Chiaradia, M. Xiloyannis, C. W. Antuvan, A. Frisoli, and L. Masia, "Design and embedded control of a soft elbow exosuit," in 2018 IEEE Int. Conf. Soft Robot. RoboSoft 2018, 2018, pp. 565-571.

[6] N. Lotti, M. Xiloyannis, G. Durandau, E. Galofaro, V. Sanguineti, L. Masia, et al., "Adaptive model-based myoelectric control for a soft wearable arm exosuit: A new generation of wearable robot control," IEEE Robot Autom. Mag., vol. 27, pp. 43-53, 2020.

[7] L. Zhang, Y. Liu, R. Wang, C. Smith, and E. M. Gutierrez-Farewik, "Modeling and Simulation of a Human Knee Exoskeleton's Assistive Strategies and Interaction," Front. Neurorobot., vol. 15, p. 13, 2021.

[8] M. Mansouri and J. A. Reinbolt, "A platform for dynamic simulation and control of movement based on OpenSim and MATLAB," $J$. Biomech., vol. 45, pp. 1517-1521, 2012.

[9] M. H. Akhavanfar, S. C. Brandon, S. H. Brown, and R. B. Graham, "Development of a novel MATLAB-based framework for implementing mechanical joint stability constraints within OpenSim musculoskeletal models," J. Biomech., vol. 91, pp. 61-68, 2019.

[10] D. G. Thelen, F. C. Anderson, and S. L. Delp, "Generating dynamic simulations of movement using computed muscle control," J. Biomech., vol. 36, pp. 321-328, 2003.

[11] K. Stollenmaier, W. Ilg, and D. F. Haeufle, "Predicting perturbed human arm movements in a neuro-musculoskeletal model to investigate the muscular force response," Front. Bioeng. Biotechnol., vol. 8, p. 308, 2020.

[12] L.-F. Lee and B. R. Umberger, "Generating optimal control simulations of musculoskeletal movement using OpenSim and MATLAB," PeerJ, vol. 4, p. e1638, 2016.

[13] D. G. Thelen, "Adjustment of muscle mechanics model parameters to simulate dynamic contractions in older adults," J. Biomech. Eng., vol. 125, pp. 70-77, 2003.

[14] N. H. Bhanpuri, A. M. Okamura, and A. J. Bastian, "Predicting and correcting ataxia using a model of cerebellar function," Brain, vol. 137, pp. 1931-1944, 2014.

[15] S. L. Delp, F. C. Anderson, A. S. Arnold, P. Loan, A. Habib, C. T. John, et al., "OpenSim: open-source software to create and analyze dynamic simulations of movement," IEEE. Trans. Biomed. Eng., vol. 54, pp. 1940-1950, 2007.

[16] A. Rajagopal, C. L. Dembia, M. S. DeMers, D. D. Delp, J. L. Hicks, and S. L. Delp, "Full-body musculoskeletal model for muscle-driven simulation of human gait," IEEE. Trans. Biomed. Eng., vol. 63, pp. 2068-2079, 2016.

[17] M. Millard, T. Uchida, A. Seth, and S. L. Delp, "Flexing computational muscle: modeling and simulation of musculotendon dynamics," $J$. Biomech. Eng., vol. 135, 2013.

[18] D. G. Lloyd and T. F. Besier, "An EMG-driven musculoskeletal model to estimate muscle forces and knee joint moments in vivo," J. Biomech., vol. 36, pp. 765-776, 2003.

[19] L. Lu, Q. Wu, X. Chen, Z. Shao, B. Chen, and H. Wu, "Development of a sEMG-based torque estimation control strategy for a soft elbow exoskeleton," Rob. Auton. Syst., vol. 111, pp. 88-98, 2019.

[20] D. G. Thelen and F. C. Anderson, "Using computed muscle control to generate forward dynamic simulations of human walking from experimental data," J. Biomech., vol. 39, pp. 1107-1115, 2006.

[21] B. R. Umberger, K. G. Gerritsen, and P. E. Martin, "A model of human muscle energy expenditure," Comput. Methods Biomech. Biomed. Eng., vol. 6, pp. 99-111, 2003. 
[22] Ž. Kozinc, J. Babič, and N. Šarabon, "Human pressure tolerance and effects of different padding materials with implications for development of exoskeletons and similar devices," Appl. Ergon., vol. 93, p. 103379, 2021.

[23] K. Huysamen, M. de Looze, T. Bosch, J. Ortiz, S. Toxiri, and L. W. O'Sullivan, "Assessment of an active industrial exoskeleton to aid dynamic lifting and lowering manual handling tasks," Appl. Ergon., vol. 68, pp. 125-131, 2018.

[24] M. B. Yandell, D. M. Ziemnicki, K. A. McDonald, and K. E. Zelik, "Characterizing the comfort limits of forces applied to the shoulders, thigh and shank to inform exosuit design," PloS one, vol. 15, p. e0228536, 2020. 


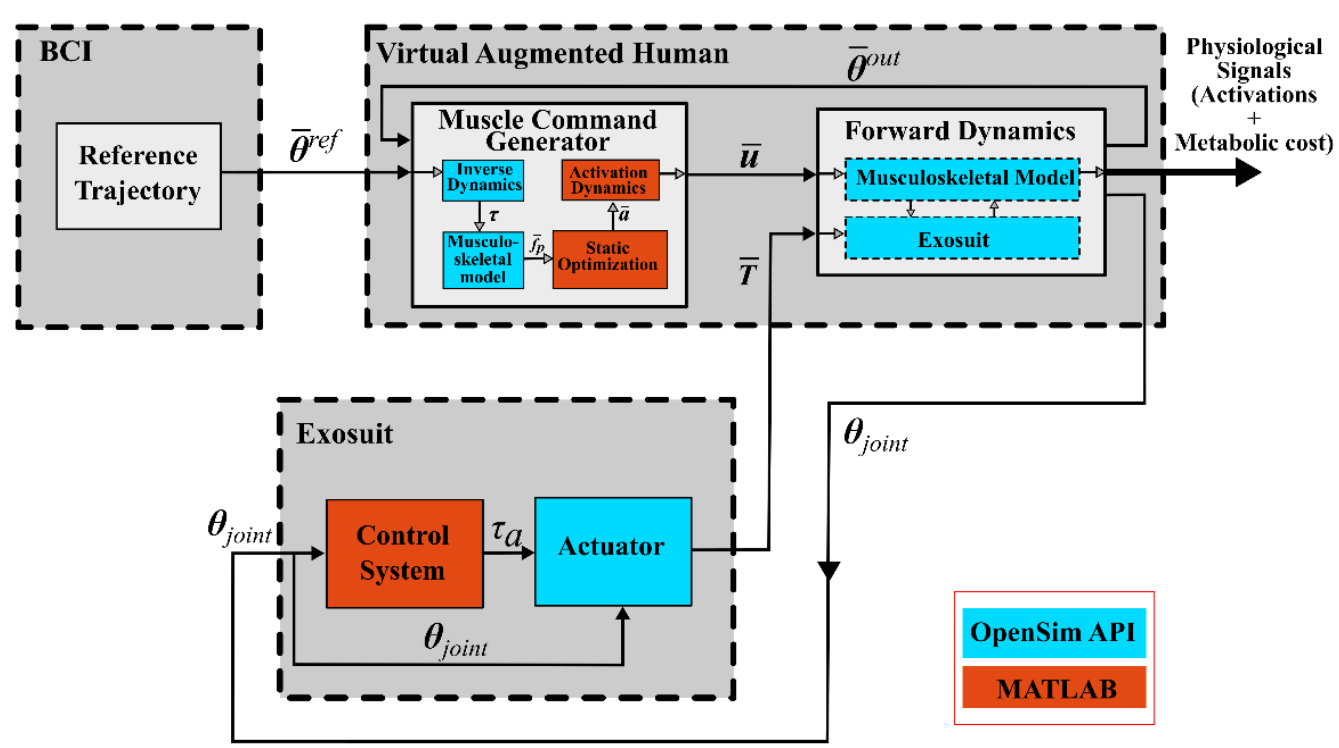

Fig.1 The overall architecture of the proposed simulation framework, where virtual human represents the user wearing the exosuit. $\theta^{\text {ref }}$ represents the reference trajectory generated by the user's mind estimated from $\mathrm{BCl} . \bar{\theta}^{\text {out }}$ : output joint kinematics, $\tau$ : joint torque, $\bar{f}_{p}$ : the input parameters for static optimization, $\bar{a}$ : muscle activations $\bar{u}$ : motor commands (muscle excitations), $\bar{T}$ : tensions in the extensor and flexor cables, $\tau_{a}$ : desired actuator torque at elbow, and $\theta_{\text {joint }}$ : output position of elbow joint. The physiological signals (muscle activations and metabolic costs) are the output of this simulation framework. 


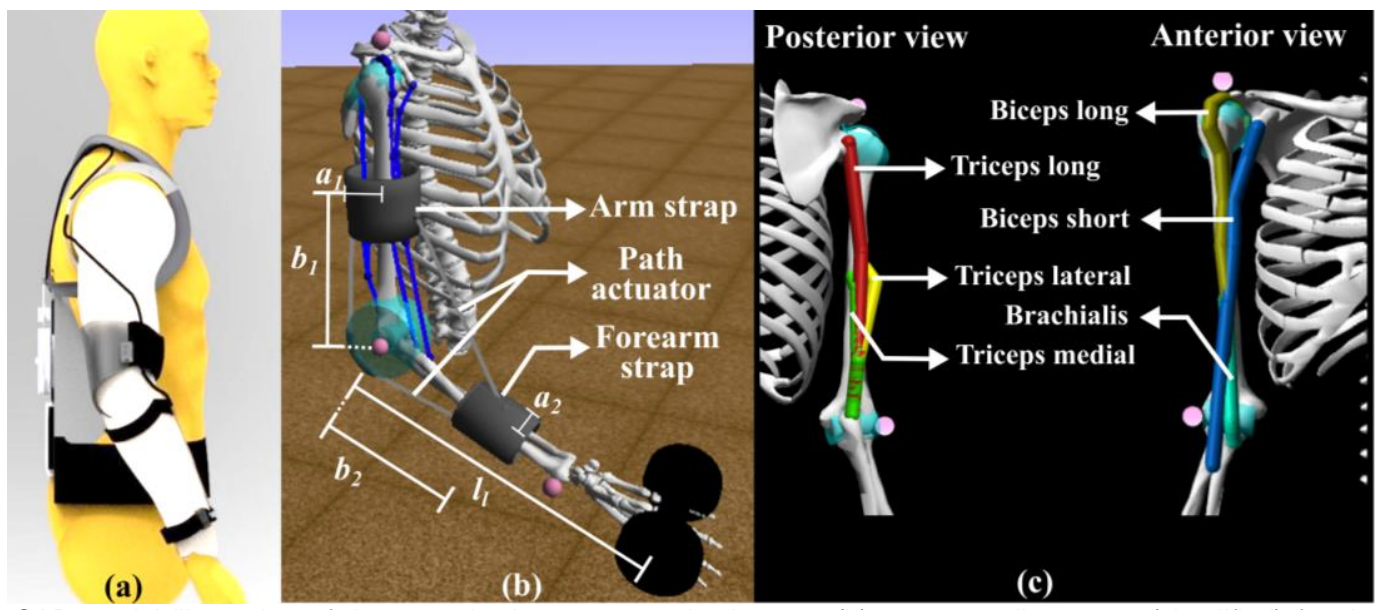

Fig.2. The (a) CAD model illustration of the exosuit placement on the human, (b) corresponding setup (simplified) for the elbow flexion simulation using the MATLAB-OpenSim framework, and (c) the muscles included in the present model. 


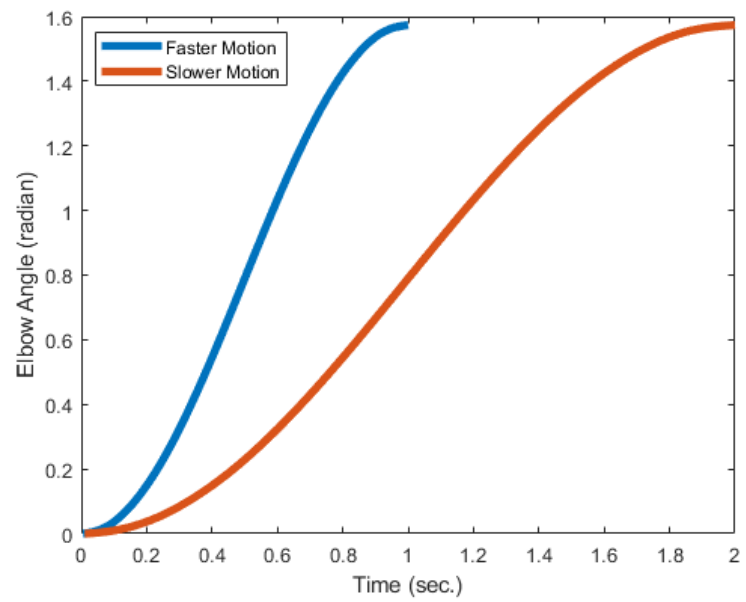

Fig. 3 - Reference Trajectories are minimum jerk trajectories from 0 to 1.57 rad in $1 \mathrm{~s}$ and $2 \mathrm{~s}$ respectively for faster and slower motion. 

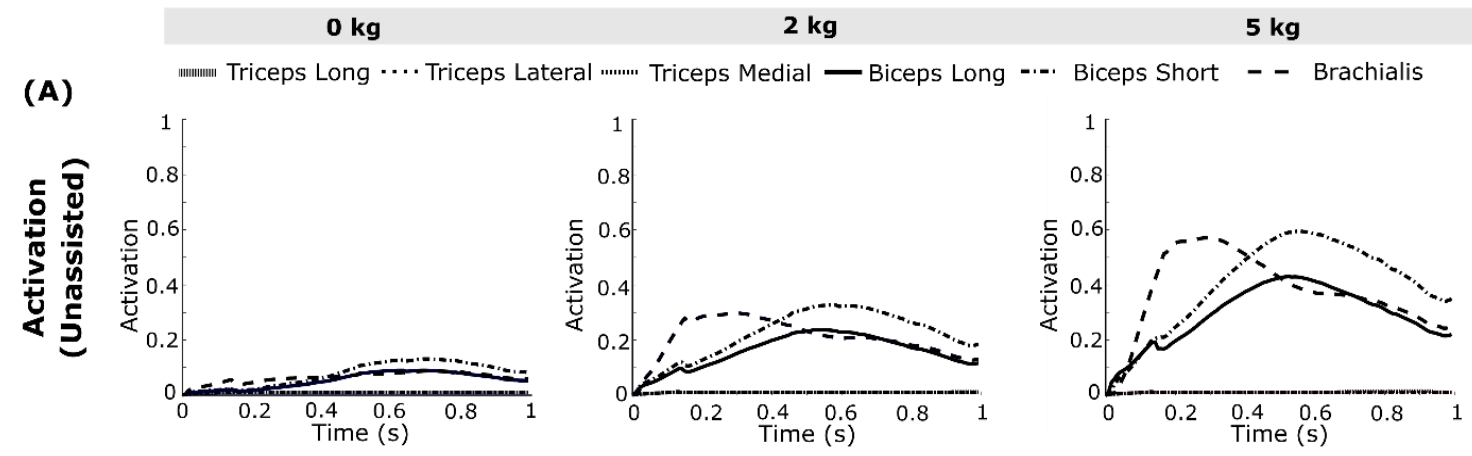

(B)
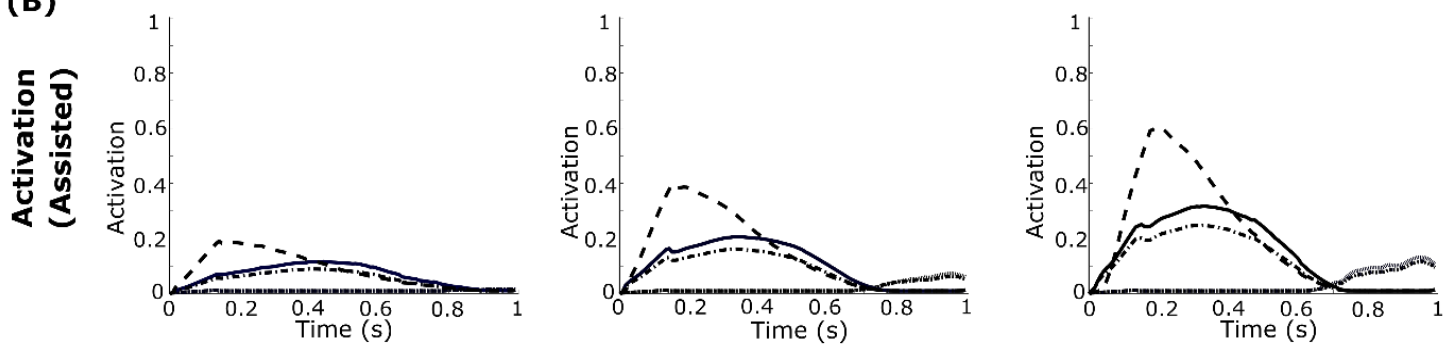

Fig. 4. The resulting activations, (A) unassisted and (B) assisted, obtained from the simulation of the framework for the elbow flexion with no load, $2 \mathrm{~kg}$ load and $5 \mathrm{~kg}$ load at the palm illustrate a visible reduction in the muscle activations after assistance from an external actuator implementing the gravity compensation control scheme. 


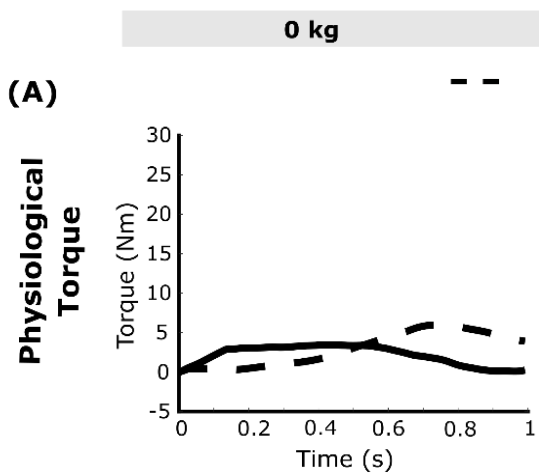

$2 \mathbf{~ k g}$

$5 \mathbf{~ k g}$

(A)

Without Assistance

- With Assistance
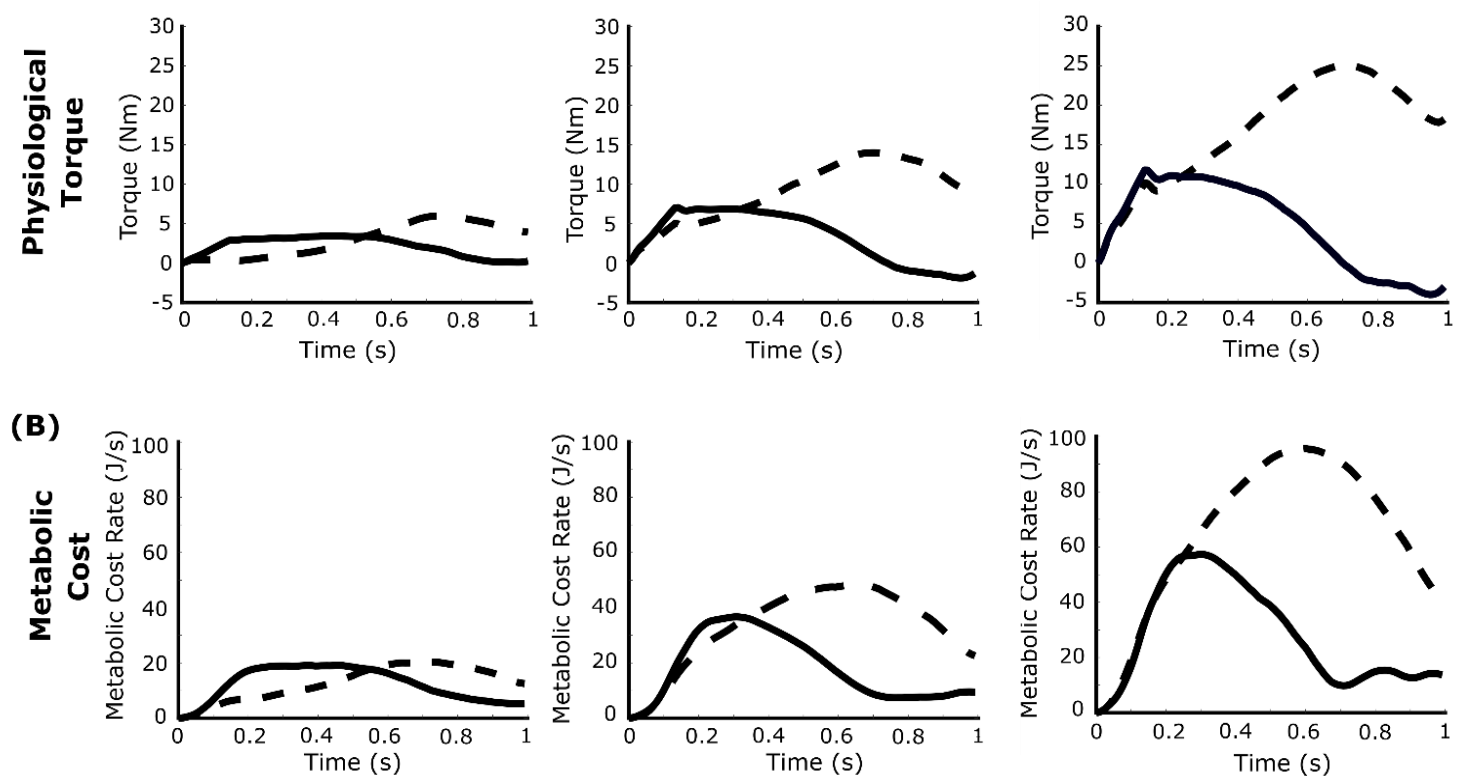

Fig. 5. The resultant (A) physiological torques about the elbow and (B) the metabolic costs calculated from the elbow flexion simulation with no load, $2 \mathrm{~kg}$ load and $5 \mathrm{~kg}$ load at the palm. The physiological torque plots (with assistance) show negative magnitude owing to the activation in the antagonistic muscles. 


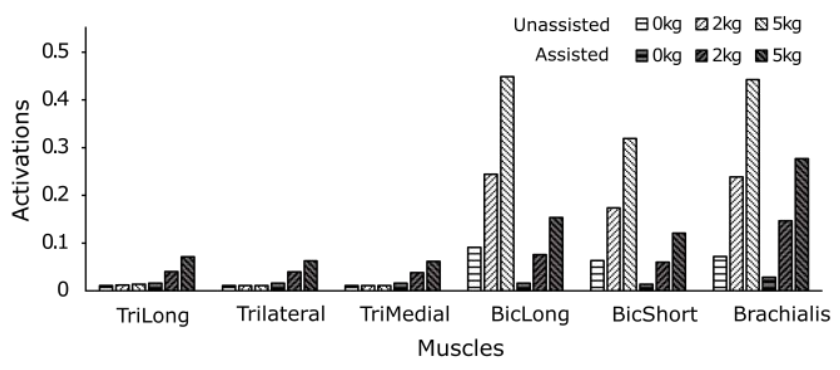

(a)

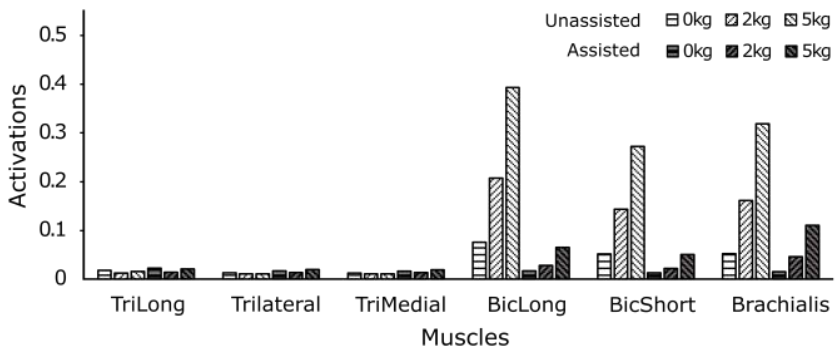

(b)

Fig. 6. The RMS values of muscle activations for (a) high speed, and (b) low speed motion with and without assistance show a trend of reduced muscle activations with external assistance. However, at higher speed of motion the triceps group presents an increase in activations, after receiving actuator assistance. 
0 kg

\section{(A) Fast Trajectory}

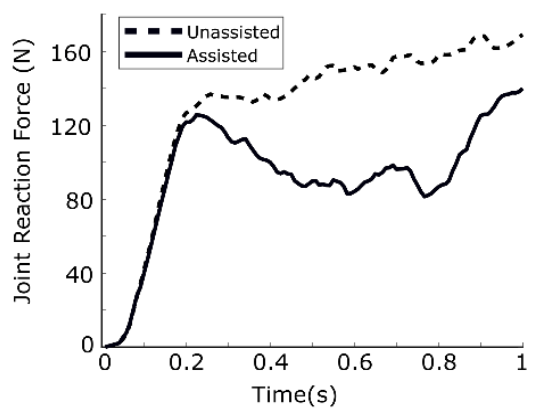

(B) Slow Trajectory
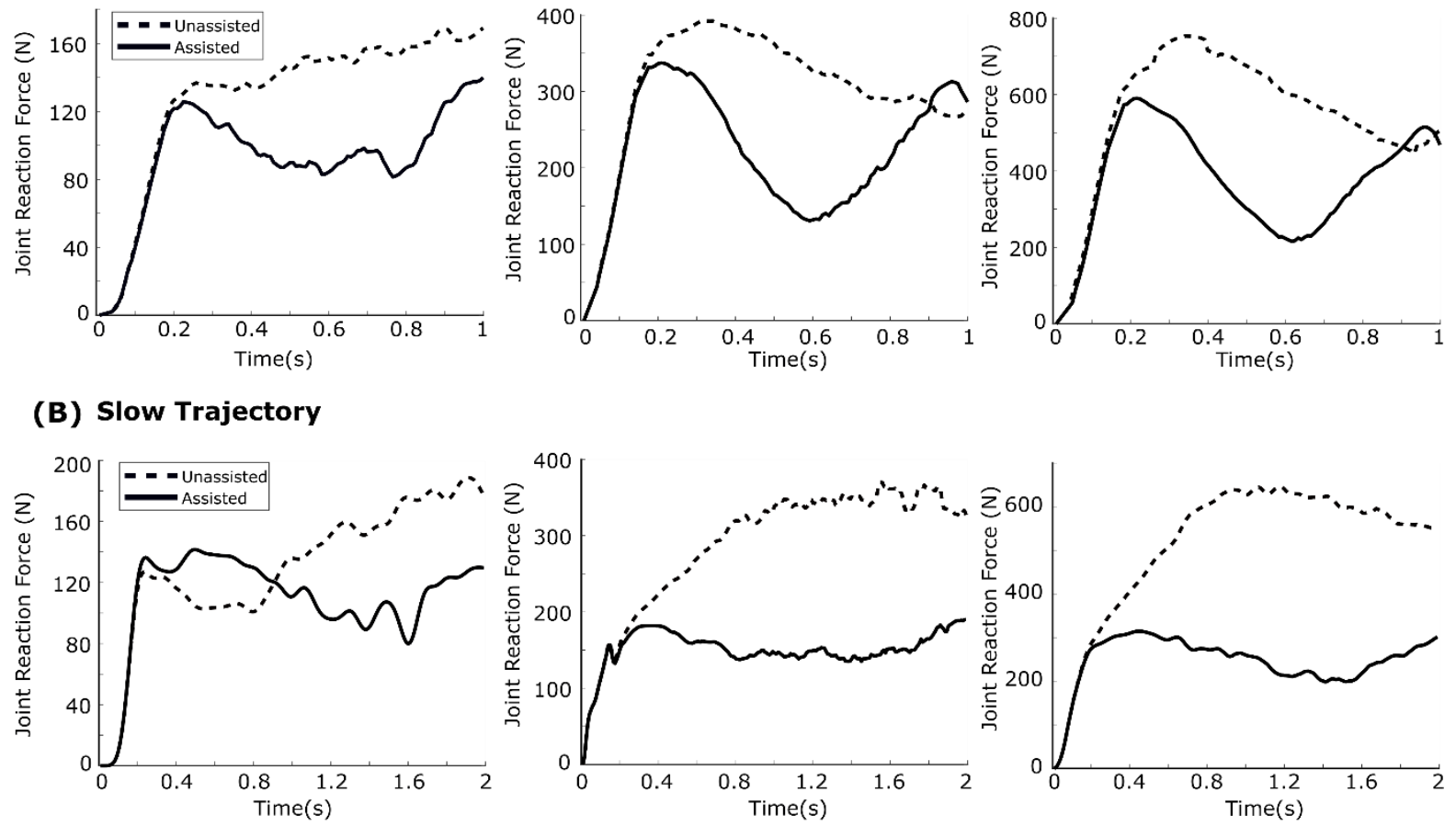

(C) Normal Force
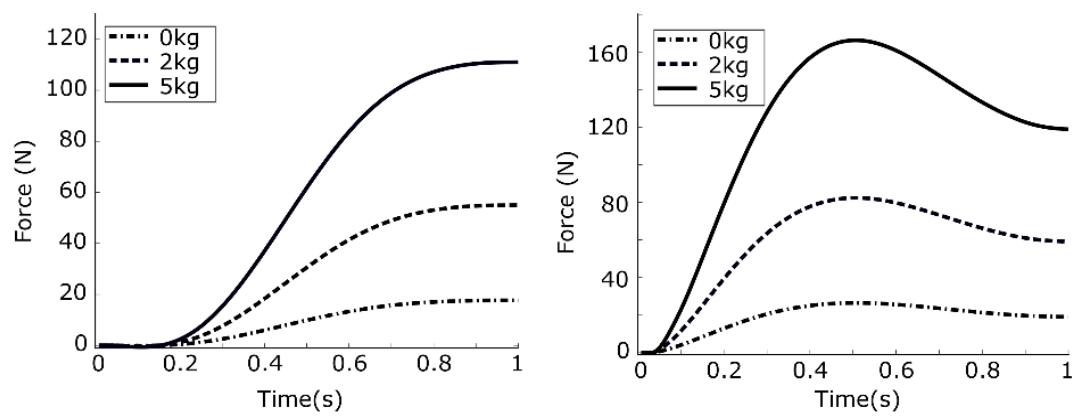

Fig. 7. The joint reaction forces calculated from the simulation framework for the $(A)$ fast trajectory and (B) slow trajectory show a trend of reduction while receiving external assistance. The reduction seems more pronounced in case of the slow speed trajectory. The (C) normal force, and (D) shear force at the forearm strap illustrate a proportionality between the external load and resultant forces. 
Table 1 - The geometrical parameters in the present model

\begin{tabular}{ccc}
\hline Parameter & Symbol & Value \\
\hline Forearm Mass & $\boldsymbol{m}$ & $1.53 \mathrm{~kg}$ \\
Forearm COM from Elbow & $\boldsymbol{l}_{\boldsymbol{c}}$ & $0.18 \mathrm{~m}$ \\
$\begin{array}{c}\text { Joint } \\
\text { Moment of inertia of forearm } \\
\text { segment about elbow joint } \\
\text { Distance of external load from } \\
\begin{array}{c}\text { Elbow Joint } \\
\text { Arm anchorage point location } \\
\text { w.r.t Elbow Joint: }\end{array}\end{array}$ & $\boldsymbol{I}_{x \boldsymbol{x}}, \boldsymbol{I}_{\boldsymbol{y} y}, \boldsymbol{I}_{z z}$ & $(0.02,0.001,0.02) \mathrm{kgm}^{2}$ \\
$\begin{array}{l}\text { Forearm anchorage point } \\
\text { location w.r.t Elbow Joint: }\end{array}$ & $\boldsymbol{a}_{\boldsymbol{l}}, \boldsymbol{b}_{\boldsymbol{1}}$ & $0.35 \mathrm{~m}$ \\
\hline & $\boldsymbol{a}_{2,}, \boldsymbol{b}_{2}$ & $(0.04 \mathrm{~m}, 0.14 \mathrm{~m})$ \\
\hline
\end{tabular}

